\title{
Development of Fault Identification System for Electric Servo Actuators of Multilink Manipulators Using Logic-Dynamic Approach
}

\author{
V. Filaretov, ${ }^{1,2}$ A. Zuev, ${ }^{1,2}$ A. Zhirabok, ${ }^{3}$ and A. Protsenko ${ }^{3}$ \\ ${ }^{1}$ Institute of Automation and Control Processes, FEB RAS, Vladivostok, Russia \\ ${ }^{2}$ Far Eastern Federal University, Vladivostok, Russia \\ ${ }^{3}$ Institute of Marine Technology Problems, Vladivostok, Russia
}

Correspondence should be addressed to A.Zuev; alvzuev@gmail.com

Received 28 April 2017; Revised 24 August 2017; Accepted 7 September 2017; Published 1 November 2017

Academic Editor: Chunhui Zhao

Copyright (C) 2017 V. Filaretov et al. This is an open access article distributed under the Creative Commons Attribution License, which permits unrestricted use, distribution, and reproduction in any medium, provided the original work is properly cited.

\begin{abstract}
This paper presents the method of synthesis of faults identification systems for electric servo actuators of multilink manipulators. These actuators are described by nonlinear equations with significantly changing coefficients. The proposed method is based on logic-dynamic approach for design of diagnostic observers for fault detection and isolation. An advantage of this approach is that it allows studying systems with nonsmooth nonlinearities by linear methods only. For solving the task of faults identification, a residual signal feedback was proposed to be used for observers. The efficiency of the proposed fault identification system was confirmed by results of simulation.
\end{abstract}

\section{Introduction}

At present, the use of multilink manipulators (MM) has considerably extended and the operations which are carried out by them become more complicated [1-4]. The occurrence of any faults in actuators of MM may lead to significant aggravation of quality of performance of the MM operations and reduction of accuracy. Therefore, the reliability and safety of such complex equipment at operating in autonomous mode have the significant importance. Today the most typical actuators of MM are electric servo actuators. One way to improve the reliability of electric servo actuators of MM is using real-time fault diagnosis and fault tolerant control [1]. Fault tolerant control is based on forming special control, providing automatic stabilization of the main parameters of actuators when faults occur [2]. For providing such control, all possible faults should be timely detected and their values should be estimated.

The problem of fault detection and isolation (FDI) was extensively investigated for the past 20 years; see, for example, the surveys $[2,5-8]$ and the books $[9,10]$. Many problems have been studied and solved: different methods of residual generation and relationships including robustness and adaptive threshold test, $H_{\infty}$-approach, and fuzzy logic; many types of systems have been considered: linear, descriptor, and different classes of nonlinear systems. Many practical examples were considered; in particular, special book was devoted to robotic systems [11].

As a rule, the electric servo actuators of MM are nonlinear essentially with such types of no differentiable nonlinearities as saturation, Coulomb friction, backlash, and hysteresis [12]. Therefore, one has to use nonlinear methods of the FDI. However, most of papers dealing with the FDI problem consider the nonlinear systems with differential nonlinearities [13-16]; therefore they cannot be used in our case.

At present, there are several approaches for designing diagnostic observers allowing fault diagnosis for systems with no differentiable nonlinearities. Among them there are methods using the algebra of functions [17], the logic-dynamic approach (LDA) [18-20], and others [21-24]. Whereas the algebra of functions demands complex analytical calculations using symbolic mathematical packages, the LDA allows 
using the methods of linear algebra for nonlinear systems and provides relatively simple procedure for synthesis of diagnostic observers. The LDA includes three steps: replacing the original nonlinear system with appropriate linear system, constructing the set of linear diagnostic observers, and adding to these observes the nonlinear parts. But LDA can solve only FDI problem without fault identification. To estimate the value of the faults, one uses in $[19,20]$ a feedback in diagnostic observers. These methods provide the faults identification for only first-order diagnostic observers. But at case when electric servo actuators of MM have partially measured state vector, then there are no guarantees that observers will have first order.

The present paper suggests a solution to the problem of developing the method of synthesis of fault identification (FI) system for electric servo actuators of MM which are described by mathematical model including differential equations with nonlinearities and partially measured state vector. The proposed method assumes the use of the diagnostic observers based on LDA with special feedback signal dependent on the residual for real-time identification of fault value.

The paper is organized as follows. Section 2 describes the model of electric servo actuators of MM. Section 3 is devoted to the synthesis of diagnostic observers for FDI using logicdynamic approach. Firstly, the main steps of this approach are given and then the observers are described in detail. In Section 4, the methods of synthesis of FI system for created observers are presented. First, the analysis of mismatch of states of electric servo actuator and the observer is given and then the synthesis of residual signal feedback is shown. An example is considered in Section 5. Section 6 concludes the paper.

\section{Description of Dynamics of Electric Servo Actuators of MM}

In this paper, the MM with PUMA type kinematic scheme with 6 degrees of freedom is considered (see Figure 1). Today, the MM with such kinematic scheme are mostly used in industry. Each degree has an electric servo actuator that includes DC motor, reducing gear and sensors measuring the electric current, angular speed of DC motor rotor, and angle of rotation of output shaft of reducing gear.

It is expedient to use the second form Lagrange equation or other methods to obtain the moment torques $M_{i}$ in each degree of freedom of the MM (see Figure 1) while moving its gripper along the complex spatial trajectory, if laws of change of generalized coordinates $q_{i}(i=\overline{1,6})$ of the MM are known.

The expressions for moments acting in each degree of freedom of MM can be represented in the form [3]

$$
M_{i}=H_{i i}(q) \ddot{q}_{i}+h_{i}(q, \dot{q}) \dot{q}_{i}+M_{E i}(q, \dot{q}, \ddot{q}),
$$

where $H_{i i}(q)$ is the diagonal element of matrix of inertia of $\mathrm{MM} ; h_{i}(q, \dot{q})$ is the element of the vector of Coriolis and centrifugal forces; and $M_{E i}(q, \dot{q}, \ddot{q})$ is the torque which represents interactions acting to $i$ th degree of freedom from other MM's links and also action of the gravitational forces. These values with the help of second Lagrange equation are defined. Further for simplicity we will not write the index $i$.

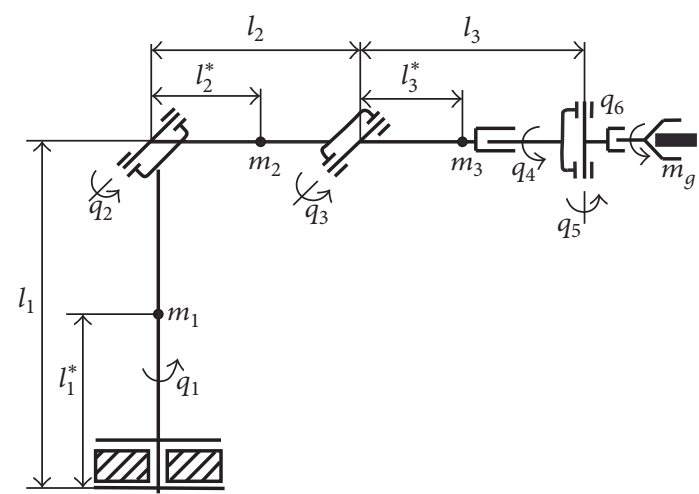

FIGURE 1: MM with PUMA type kinematic scheme.

It is assumed that the following typical faults are possible in each electric servo actuator of the MM:

(i) The fault $d_{1}(t)$ caused by change of value of Coulomb friction moment $\widetilde{M}_{\mathrm{cfg} 0}$ in reducing gear

(ii) The fault $d_{2}(t)$ caused by change of value of Coulomb friction moment $\widetilde{M}_{\mathrm{cfm} 0}$ in DC motor

(iii) The fault $d_{3}(t)$ caused by change of value of electrical resistance $\widetilde{R}$.

Considering (1) and these faults, model of electric servo actuator of the MM can be presented by the following differential equations:

$$
\begin{aligned}
\dot{x}_{1}= & x_{2}, \\
\dot{x}_{2}= & -\frac{c i_{g}{ }^{2}}{H(q)} x_{1}-\frac{\left(k_{v g}+h(q, \dot{q})\right)}{H(q)} x_{2}-\frac{M_{E}(q, \dot{q}, \ddot{q})}{H(q)} \\
& -\frac{M_{\mathrm{cfg} 0}}{H(q)} \operatorname{sign}\left(x_{2}\right)+d_{1}(t), \\
\dot{x}_{3}= & x_{4}, \\
\dot{x}_{4}= & \frac{c i_{g}}{J} x_{1}-\frac{c}{J} x_{3}-\frac{k_{v m}}{J} x_{4}+\frac{k_{m}}{J} x_{5} \\
& -\frac{M_{\mathrm{cfm} 0}}{J} \operatorname{sign}\left(x_{4}\right)+d_{2}(t), \\
\dot{x}_{5}= & \frac{-k_{\omega}}{L} x_{4}-\frac{R}{L} x_{5}+\frac{k_{u}}{L} u+d_{3}(t),
\end{aligned}
$$

where $x_{1}=\dot{a}_{g}$ and $x_{2}=a_{g}$ are speed and angle of output shaft of reducing gear; $x_{3}=\dot{a}_{m}$ and $x_{4}=a_{m}$ are speed and angle of DC motor shaft; $R, L$, and $x_{5}=I$ are resistance, inductance, and current of electric motor rotor circuits, accordingly; $k_{\omega}$ is a coefficient of counter-EMF; $k_{m}$ is a coefficient of motor moment; $J$ is a rotational inertia of rotating parts of reduction drive and DC motor; $c$ is rigidity of mechanical transmission; $i_{g}$ is a coefficient of the reducing gear; $u$ is control signal; $k_{u}$ is an amplifier gain; $M_{\mathrm{cfm} 0}$ and $M_{\mathrm{cfg} 0}$ are coefficients of 
Coulomb friction of DC motor and reducing gear; and the variables $d_{j}(j=1,2,3)$ represent the effects of faults on the system and are of the form

$$
\begin{aligned}
& d_{1}(t)=-\frac{\widetilde{M}_{\mathrm{cfg} 0}}{H(q)} \operatorname{sign}\left(x_{2}\right), \\
& d_{2}(t)=-\frac{\widetilde{M}_{\mathrm{cfm} 0}}{H(q)} \operatorname{sign}\left(x_{2}\right), \\
& d_{3}(t)=-\frac{\widetilde{R}}{L} I .
\end{aligned}
$$

Equation (2) has nonlinearities and significantly changing coefficients because of changes in parameters $H(q), h(q, \dot{q})$, and $M_{E}(q, \dot{q}, \ddot{q})$.

\section{Synthesis of Diagnostic Observers for FDI Using LDI}

System (2) can be represented by the differential equation in a matrix form as follows:

$$
\begin{aligned}
& \dot{x}(t)=F x(t)+G u(t)+B(x)+E d(t), \\
& y(t)=C x(t),
\end{aligned}
$$

where $B(x)$ is a vector corresponding to the system nonlinearities, $x \in R^{5}, y \in R^{3}, C \in R^{3 \times 5}, F \in R^{5 \times 5}, G \in R^{5}$,

$$
\begin{aligned}
& F \\
& =\left[\begin{array}{ccccc}
0 & 1 & 0 & 0 & 0 \\
-\frac{c i_{g}^{2}}{H(q)} & -\frac{\left(k_{v g}+h(q, \dot{q})\right)}{H(q)} & \frac{c i_{g}}{H(q)} & 0 & 0 \\
0 & 0 & 0 & 1 & 0 \\
\frac{c i_{g}}{J} & 0 & -\frac{c}{J} & -\frac{k_{v m}}{J} & \frac{k_{m}}{J} \\
0 & 0 & 0 & -\frac{k_{\omega}}{L} & -\frac{R}{L}
\end{array}\right],
\end{aligned}
$$

$$
\begin{aligned}
& E=\left[\begin{array}{lll}
0 & 0 & 0 \\
1 & 0 & 0 \\
0 & 0 & 0 \\
0 & 1 & 0 \\
0 & 0 & 1
\end{array}\right], \\
& C=\left[\begin{array}{lllll}
1 & 0 & 0 & 0 & 0 \\
0 & 0 & 0 & 1 & 0 \\
0 & 0 & 0 & 0 & 1
\end{array}\right],
\end{aligned}
$$

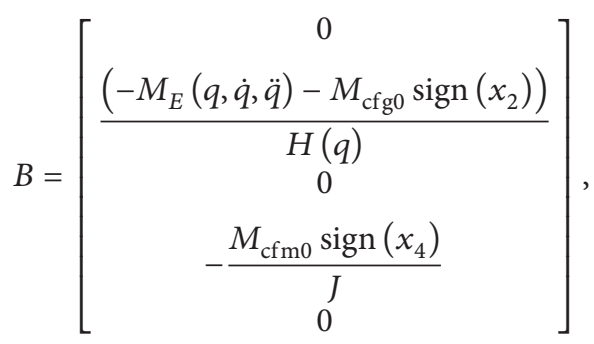

$x=\left[\begin{array}{c}a_{g} \\ \dot{a}_{g} \\ a_{m} \\ \dot{a}_{m} \\ I\end{array}\right]$,

$G=\left[\begin{array}{c}0 \\ 0 \\ 0 \\ 0 \\ \frac{k_{u}}{L}\end{array}\right]$.

If faults occur during the work of MM, they should be promptly detected and identified. Then the fault tolerant control can be used. The traditional methods of diagnosis that focus on systems described by linear models cannot provide the solution to the problem of synthesis of FI systems for electric servo actuators of the MM described by model with the parameters $H(q), h(q, \dot{q})$, and $M_{E}(q, \dot{q}, \ddot{q})$ that are significantly variable and under the presence of the nonlinear component $B(x)$.

In this paper, another synthesis method of FI systems is proposed. This method includes two basic steps [25]: (1) using LDA to construct the diagnostic observer $[17,18]$ which can detect occurrence of specific faults; (2) introducing the specific feedback in observers for identification of the fault values [19]. Below these stages are considered in more detail.

The LDA to solve the FDI problem for system (4) includes the following steps [18]: (1) replacing the initial nonlinear system (4) by linear system; (2) solving the FDI problem for the linear system with some additional restrictions and obtaining the bank of linear observers; (3) transforming the linear observers into the nonlinear ones. Thus, the nonlinear diagnostic observers detecting the faults are of the following general form:

$$
\begin{aligned}
& \dot{x}_{*}(t)=F_{*} x_{*}(t)+G_{*} u(t)+B_{*}\left(x_{*}\right)+M_{*} y(t), \\
& y_{*}(t)=C_{*} x_{*}(t),
\end{aligned}
$$

where $x_{*}(t) \in R^{k}$ is the state vector of the observer, $y_{*}(t)$ is the output vector, $F_{*} \in R^{k \times k}, G_{*} \in R^{k}, B_{*} \in R^{k}, M_{*} \in R^{k \times 3}$, and $C_{*} \in R^{k}$ are constant matrices, and $k$ is dimension of the 
observer; it is assumed that the matrices $F_{*}$ and $C_{*}$ can be represented in a canonical form

$$
\begin{aligned}
& F_{*}=\left[\begin{array}{lllll}
0 & 1 & 0 & \cdots & 0 \\
0 & 0 & 1 & \cdots & 0 \\
& \cdot & \cdot & \cdot & \\
0 & 0 & 0 & \cdots & 0
\end{array}\right], \\
& C_{*}=\left[\begin{array}{lllll}
1 & 0 & 0 & \cdots & 0
\end{array}\right] .
\end{aligned}
$$

The observer (6) generates the residual $r(t)=N y(t)-$ $y_{*}(t)$ for certain row matrix $N \in R^{3}$ which has to be determined. If there are no faults and $d(t)=0$, then $r(t)=0$; if a fault occurs, $r(t) \neq 0$. It is well-known from the linear FDI theory $[2,9,26]$ that, for the observer design, the matrix $\Phi$ where $\Phi x(t)=x_{*}(t)$ in the unfaulty case after the response to unlike conditions has died out plays the main role. In the absence of faults, the following well-known set of equations holds $[2,9,26]$ :

$$
\begin{aligned}
C_{*} \Phi & =N C, \\
\Phi F & =F_{*} \Phi+M_{*} C, \\
G_{*} & =\Phi G, \\
B_{*}\left(x_{*}\right) & =\Phi B(x) .
\end{aligned}
$$

For each fault $d_{i}$, the diagnostic observer $O_{i}$ should be constructed. Matrices and vectors $\Phi, F_{*}, B_{*}\left(x_{*}\right), G_{*}, N$, and $M_{*}$ describing the observer can be found in (8) [17]. As a result, the observers $O_{1}, O_{2}$, and $O_{3}$ for the faults $d_{1}, d_{2}$, and $d_{3}$ were obtained:

$$
\mathrm{O}_{1} \text { : }
$$

$$
\begin{gathered}
\Phi=\left[\begin{array}{ccccc}
1 & 0 & 0 & 0 & 0 \\
\frac{\left(k_{v g}+h(q, \dot{q})\right)}{H(q)} & 1 & 0 & 0 & 0 \\
0 & 0 & \frac{c i_{g}}{H(q)} & 0 & 0
\end{array}\right], \\
M_{*}=\left[\begin{array}{ccc}
-\frac{\left(k_{v g}+h(q, \dot{q})\right)}{H(q)} & 0 & 0 \\
-\frac{c i_{g}^{2}}{H(q)} & 0 & 0 \\
0 & \frac{c i_{g}}{H(q)} & 0
\end{array}\right], \\
N=\left[\begin{array}{lll}
1 & 0 & 0
\end{array}\right],
\end{gathered}
$$

$$
F_{*}=\left[\begin{array}{lll}
0 & 1 & 0 \\
0 & 0 & 1 \\
0 & 0 & 0
\end{array}\right]
$$$$
G_{*}=\left[\begin{array}{l}
0 \\
0 \\
0
\end{array}\right],
$$$$
B_{*}=\left[\begin{array}{c}
0 \\
-\frac{M_{E}(q, \dot{q}, \ddot{q})+M_{\mathrm{cfg} 0} \operatorname{sign}\left(x_{2}\right)}{H(q)} \\
0
\end{array}\right] .
$$

$\mathrm{O}_{2}$ :

$$
\begin{aligned}
& \Phi=\left[\begin{array}{ccccc}
0 & 0 & 0 & 1 & 0 \\
0 & 0 & -\frac{c}{J} & 0 & 0
\end{array}\right], \\
& M_{*}=\left[\begin{array}{rrr}
\frac{c i_{g}}{J} & -\frac{k_{v m}}{J} & \frac{k_{m}}{J} \\
0 & -\frac{c}{J} & 0
\end{array}\right] \text {, } \\
& N=\left[\begin{array}{lll}
0 & 1 & 0
\end{array}\right] \text {, } \\
& F_{*}=\left[\begin{array}{ll}
0 & 1 \\
0 & 0
\end{array}\right] \text {, } \\
& G_{*}=\left[\begin{array}{l}
0 \\
0
\end{array}\right], \\
& B_{*}=\left[\begin{array}{c}
-\frac{M_{\mathrm{cfm} 0} \operatorname{sign}\left(x_{4}\right)}{J} \\
0
\end{array}\right] .
\end{aligned}
$$

$\mathrm{O}_{3}$ :

$$
\begin{aligned}
& \Phi=\left[\begin{array}{lllll}
0 & 0 & 0 & 0 & 1
\end{array}\right], \\
& M_{*}=\left[\begin{array}{lll}
0 & -\frac{k_{\omega}}{L} & -\frac{R}{L}
\end{array}\right] \text {, } \\
& N=\left[\begin{array}{lll}
0 & 0 & 1
\end{array}\right] \text {, } \\
& F_{*}=0 \text {, } \\
& G_{*}=\frac{k_{u}}{L}, \\
& B_{*}=0 \text {. }
\end{aligned}
$$

As a result, the synthesized observers $\mathrm{O}_{1}, \mathrm{O}_{2}$, and $\mathrm{O}_{3}$ provide solving the FDI problem for electric servo actuators 
of MM. Clearly, the observers $\mathrm{O}_{1}$ and $\mathrm{O}_{2}$ have order more than one. This is caused by the fact that the electric servo actuator (2) has the partially measured state vector. Therefore, the known methods $[19,20]$ for FI cannot be used. Below the new synthesis method of FI systems for observers constructed by using LDA is proposed.

\section{Synthesis of FI System for Observers Created by Using LDA}

To solve the problem of FI for synthesized observers, consider how residual $r(t)$ changes when the faults $d_{j}(t)$ occur. After differentiating the equation for residual and taking into account (4), (6), and (8), the following can be obtained:

$$
\begin{aligned}
& \dot{r}(t)=C_{*}\left(F_{*}\left(\Phi x(t)-x_{*}(t)\right)+\Phi B(x)-B_{*}\left(x_{*}\right)\right. \\
& \quad+\Phi E d(t)) .
\end{aligned}
$$

The vector $e(t) \in R^{k}$ is introduced which is a mismatch of states of electric servo actuator and the observer:

$$
e(t)=\Phi x(t)-x_{*}(t)
$$

Taking into account the vector $C_{*}$, the residual $r(t)$ is a first element of mismatch vector $e(t): r(t)=e_{1}(t)$. Therefore, (12) can be rewritten as

$$
\dot{e}(t)=F_{*} e(t)+\Phi B(x)-B_{*}\left(x_{*}\right)+\Phi E d(t) .
$$

Analysis of (14) shows that if order of the obtained observer is one (i.e., $y_{*}(t)=x_{*}(t)$ ), then if faults occur, the following conditions are true: (1) $F_{*}=0$, (2) $e(t)=$ $r(t) \in R^{1}$, and (3) $B_{*}=\Phi B$. In this case, the feedback is proportional to the residual signal as $\operatorname{Tr}(t)$ ( $T$ is the feedback coefficient) and allows identifying the faults value $[19,20]$. However, in case when order of the observer is more than one, $B_{*} \neq \Phi B$ due to $x_{*}(t) \neq \Phi x(t)$. Moreover, in (6) the components $F_{*} e(t)$ and $\Phi B(x(t))$ are unknown. As a result, it is impossible to make fault identification. To solve this problem, one proposes a feedback of residual signal which provides $e(t)=0$. In this case the value of this feedback is proportional to the value of fault. This provides a possibility of identifying the fault value.

After the introduction of proposed residual signal feedback, the model of observer will be

$$
\begin{aligned}
\dot{x}_{*}(t)= & F_{*} x_{*}(t)+G_{*} u(t)+B_{*}\left(x_{*}\right)+M_{*} y(t) \\
& +w(r), \\
y_{*}(t)= & C_{*} x_{*}(t),
\end{aligned}
$$

where $w(r) \in R^{k}$ is the vector specifying feedback dependent on the residual signal.

Considering the feedback (15), equation for the mismatch vector is as follows:

$$
\dot{e}(t)=F_{*} e(t)+\Phi B(x)-B_{*}\left(x_{*}\right)+\Phi E d(t)-w(r) .
$$

Equation (17) can be rewritten in the form

$$
\begin{aligned}
& \dot{e}_{1}(t)=e_{2}(t)-w_{1}(r), \\
& \dot{e}_{2}(t)=e_{3}(t)-w_{2}\left(r_{1}\right), \\
& \dot{e}_{3}(t)=e_{4}(t)-w_{3}(r),
\end{aligned}
$$

$$
\begin{gathered}
\dot{e}_{v}(t)=e_{v+1}(t)+f_{v} d_{j}(t)+a_{v}-w_{v}(r) . \\
\vdots \\
\dot{e}_{k}(t)=-w_{k}(r),
\end{gathered}
$$

where $a_{v}$ is the $v$ th element of the vector $\Phi B(x(t))-B_{*}\left(x_{*}(t)\right)$, $v=\overline{1, k}$, and $f_{v}$ is the $v$ th element of vector $\Phi L$.

When $w_{v+1}(r)=0, \ldots, w_{k}(r)=0$ and the initial conditions of the electric servo actuator and the observer are agreed upon, $e_{v+1}(t), \ldots, e_{k}(t)$ are zero regardless of occurrence of the fault. Taking this into account, it is possible to rewrite (18) in the form of a differential equation:

$$
\begin{aligned}
f_{v} d_{j}(t)+a_{v}= & \dot{e}_{v}(t)+w_{v}(r) \\
= & \ddot{e}_{v-1}(t)+\dot{w}_{v-1}(r)+w_{v}(r) \\
= & \dddot{e}_{v-2}(t)+\ddot{w}_{v-2}(r)+\dot{w}_{v-1}(r)+w_{v}(r) \\
= & e_{1}^{(v)}(t)+w_{1}{ }^{(v-1)}(r)+\cdots+w^{(1)}{ }_{v-1}(r) \\
& +w_{v}(r) .
\end{aligned}
$$

Making elements of the feedback vector, except the $v$ th element, proportional to the residual $w_{n}(r)=\left(T_{n} / T_{0}\right) r(t)$ $(n=\overline{1, v-1})$ and $w_{v}(r)=\left(T_{v} / T_{0}\right) r(t)+\left(1 / T_{0}\right) z(r)\left(T_{0}, \ldots, T_{v}\right.$ are feedback coefficients), one obtains

$$
\begin{aligned}
f_{v} d_{j}(t)+a_{v}= & r^{(v)}(t)+\frac{T_{1}}{T_{0}} r^{(v-1)}(t)+\cdots \\
& +\frac{T_{v-1}}{T_{0}} r^{(1)}(t)+\frac{T_{v}}{T_{0}} r(t)+\frac{1}{T_{0}} z(r) .
\end{aligned}
$$

Feedback coefficients $T_{0}, \ldots, T_{v}$ are chosen to guarantee the stability of observers [25].

Consider $f_{v} d_{j}(t)+a_{v}$ as input signal and $r(t)$ as output; then (20) can be presented by a transfer function $W=$ $T_{0} /\left(T_{0} s^{v}+\cdots+T_{v}+z(s)\right)$, where $s$ is the symbol of Laplace transformation. Suppose that input $f_{v} d_{j}(t)+a_{v}$ can be presented by a polynomial of degree $q: f_{v} d_{j}(t)+a_{v}=b_{0}+$ $b_{1} t+\cdots+b_{q} t^{q}$. The Laplace transformation of such input is $Q=$ $b_{0} / s+b_{1} / s^{2}+\cdots+b_{q} / s^{q+1}$. Then the steady value of $r(t)$ can be found as $r(t)=\lim _{s \rightarrow 0}=\lim _{s \rightarrow 0} W Q s=T_{0}\left(b_{0} s^{q-1}+\cdots+\right.$ $\left.b_{q}\right) s /\left(\left(T_{0} s^{v}+\cdots+T_{v}\right) s^{q+1}+z(s) s^{q+1}\right)$. Assuming $z(s)=T_{v+1} / s+$ $T_{v+2} / s^{2}+\cdots+T_{v+q} / s^{q}+T_{v+q+1} / s^{q+1}$, it is possible to obtain 
$r(t)=\lim _{s \rightarrow 0}\left(T_{0}\left(b_{0} s^{q-1}+\cdots+b_{q}\right) s /\left(\left(T_{0} s^{v}+\cdots+T_{v}\right) s^{q+1}+\right.\right.$ $\left.\left.T_{v+1} s^{q}+\cdots+T_{v+q+1}\right)\right)=0$.

Thus, the introduction of such a feedback results in a steady value of $r(t)=0$. This also results in resetting all elements of the vector $e(t)$. For many real systems, value of the fault is constant or slowly changes and it is sufficient to introduce one integral of residual in feedback to achieve the required accuracy of the faults identification: $z(r)=\int r(t) d t$. Thus, after using this kind of feedback, residual tends to zero, after the completion of the transition process, including the event when the faults occur. This ensures the synchronization of state vectors of the electric servo actuator and the observer. Thus, fault $d_{j}$ can be found as

$$
d_{j}(t)=\frac{1}{f_{v} T_{0}} \int r(t) d t
$$

After applying the proposed procedure for observers $\mathrm{O}_{1}, \mathrm{O}_{2}$, and $\mathrm{O}_{3}$, we receive the following feedback for fault identification:

$$
\begin{aligned}
O_{1}: & \\
w & =\left[\begin{array}{lll}
w_{11} & w_{12} & 0
\end{array}\right]^{T}, \\
w_{11} & =\frac{T_{1}}{T_{01}} r(t), \\
w_{12} & =\frac{T_{2}}{T_{01}} r(t)+\int \frac{1}{T_{01}} r(t) d t .
\end{aligned}
$$

$\mathrm{O}_{2}$ :

$$
\begin{aligned}
w & =\left[\begin{array}{ll}
w_{12} & 0
\end{array}\right]^{T} \\
w_{12} & =\frac{T_{3}}{T_{02}} r(t)+\int \frac{1}{T_{02}} r(t) d t .
\end{aligned}
$$

$\mathrm{O}_{3}$ :

$$
w(r)=T_{4} r(t)
$$

Finally, considering the expression (21), it is possible to identify the faults value for the electric servo actuators of the MM:

$$
\begin{aligned}
\widetilde{M}_{\mathrm{cfg} 0} & =-\frac{H(q) \operatorname{sign}\left(\dot{a}_{g}\right)}{f_{v} T_{0}} \int r(t) d t, \\
\widetilde{M}_{\mathrm{cfm} 0} & =-\frac{J \operatorname{sign}\left(\dot{a}_{m}\right)}{f_{v} T_{0}} \int r(t) d t, \\
\widetilde{R} & =-\frac{r(t) L T_{4}}{I} .
\end{aligned}
$$

Thus, the problem of identification of value of faults has been solved.

\section{Simulation of Performance of FI System}

Analysis of effectiveness of developed FI system was implemented using PUMA type of the MM. The FI system was synthesized for electric servo actuator of second degree of freedom of MM (see Figure 1). The results for other degrees of freedom of the MM are similar.

The second degree of freedom of MM has the terms $H, h$, and $M_{E}$ as follows:

$$
\begin{aligned}
H & =J_{n 2}+J_{n 3}+m_{2} l_{2}^{* 2}+m_{3} l_{3}^{* 2}+\left(m_{3}+m_{g}\right) l_{2}^{2} \\
& +m_{g} l_{3}^{2}+2 l_{2}\left(m_{3} l_{3}^{*}+m_{g} l_{3}\right) \cos q_{3}, \\
h= & -2 l_{2}\left(m_{3} l_{3}^{*}+m_{g} l_{3}\right) \dot{q}_{3} \sin q_{3}, \\
M_{E}= & {\left[J_{n 3}+m_{3} l_{3}^{* 2}+m_{g} l_{3}^{2}+\left(m_{3} l_{3}^{*}+m_{g} l_{3}\right) l_{2} \cos q_{3}\right] } \\
& \cdot \ddot{q}_{3}-l_{2}\left(m_{3} l_{3}^{*}+m_{g} l_{3}\right) \dot{q}_{3}^{2} \sin q_{3}+g\left[m_{2} l_{2}^{*}\right. \\
& \left.+\left(m_{3}+m_{g}\right) l_{2}\right] \sin q_{2}+g\left(m_{3} l_{3}^{*}+m_{g} l_{3}\right) \\
& \cdot \sin \left(q_{2}+q_{3}\right) \\
& -\frac{1}{2}\left[\left(J_{n 2}-J_{s 2}+m_{2} l_{2}^{* 2}+\left(m_{3}+m_{g}\right) l_{2}^{2}\right) \sin 2 q_{2}\right. \\
& +\left(J_{n 3}-J_{s 3}+m_{3} l_{3}^{* 2}+m_{g} l_{3}^{2}\right) \sin 2\left(q_{2}+q_{3}\right) \\
& \left.+2 l_{2}\left(m_{3} l_{3}^{*}+m_{g} l_{3}\right) \sin \left(2 q_{2}+q_{3}\right)\right] \dot{q}_{1}^{2},
\end{aligned}
$$

where $J_{s i}$ and $J_{n i}$ are the moments of inertia of link $i$ along its longitudinal and transverse axis passing through its center of mass, accordingly; $m_{i}$ is the mass of link $i ; m_{g}$ is the mass of the load; $l_{i}$ is the length of link $i ; l_{i}^{*}$ is the length from $i$ th joint to the center of mass of $i$ th link; $g$ is the acceleration of gravity; and $q_{i}, \dot{q}_{i}, \ddot{q}_{i}$ are the angle of rotation of the link $i$ and its derivatives.

The following set of parameters of MM was used for simulation: $l_{1}=l_{2}=l_{3}=0.5 \mathrm{~m}, l_{1}^{*}=0.1 \mathrm{~m} ; l_{2}^{*}=l_{3}^{*}=0.2 \mathrm{~m}$, $m_{g}=5 \mathrm{~kg} ; m_{1}=25 \mathrm{~kg}, m_{2}=m_{3}=5 \mathrm{~kg} ; J_{s 2}=0.007 \mathrm{~kg} \cdot \mathrm{m}^{2}$, $J_{s 3}=0.005 \mathrm{~kg} \cdot \mathrm{m}^{2}, J_{n 2}=0.14 \mathrm{~kg} \cdot \mathrm{m}^{2}, J_{n 3}=0.2 \mathrm{~kg} \cdot \mathrm{m}^{2}, R=$ $0.5 \Omega, L=0.0005 \mathrm{H}, k_{\omega}=0.04 \mathrm{~V} \cdot \mathrm{s}, k_{m}=0.04 \mathrm{~N} \cdot \mathrm{m} / \mathrm{A}, J=$ $10^{-3} \mathrm{~kg} \cdot \mathrm{m}^{2}, i_{g}=100 ; k_{u}=800, M_{\mathrm{cfg} 0}=M_{\mathrm{cfm} 0}=0.02 \mathrm{~N} \cdot \mathrm{m}$, and $k_{v g}=k_{v m}=0.005 \mathrm{~N} \cdot \mathrm{m} \cdot \mathrm{s} / \mathrm{rad}$.

The input signals $q_{1}=\sin (0.2 t), q_{2}=u=\sin (0.5 t)$, and $q_{3}=\sin (t)$ were applied to the electric servo actuator. The parameters of feedback for observers were chosen as follows:

$$
\begin{aligned}
& T_{01}=1.372 \times 10^{-6}, \\
& T_{02}=2.564 \times 10^{-4}, \\
& T_{1}=3.704 \times 10^{-4}, \\
& T_{2}=0.033, \\
& T_{3}=0.033, \\
& T_{4}=100 .
\end{aligned}
$$




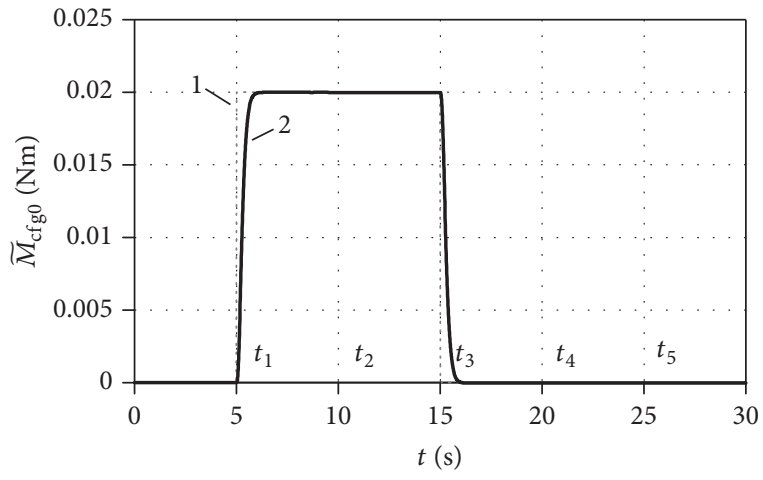

FIGURE 2: Estimation of constant fault $\widetilde{M}_{\mathrm{cfg} 0}$ value by proposed FI system.

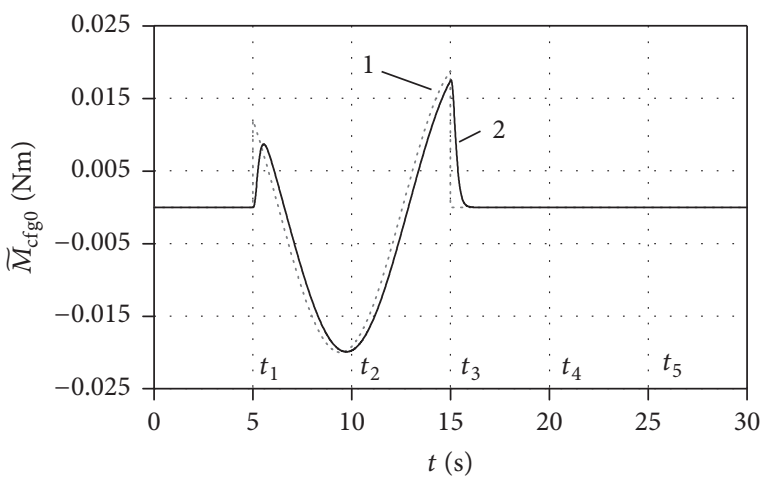

Figure 3: Estimation of variable fault $\widetilde{M}_{\mathrm{cfg} 0}$ value by proposed FI system.

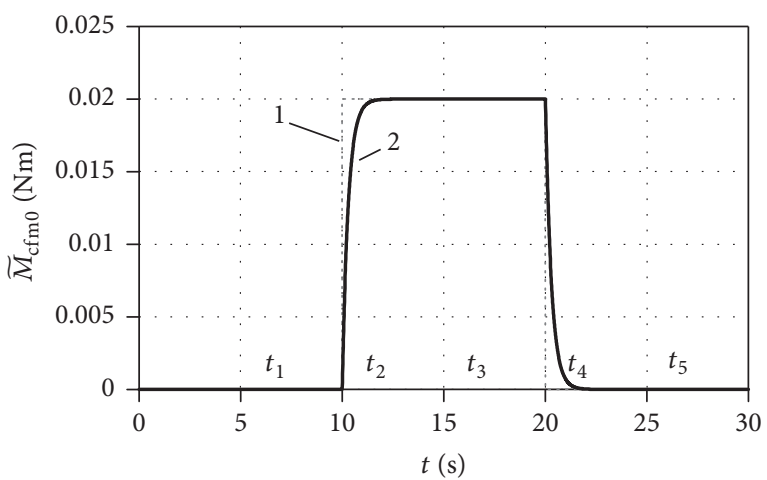

Figure 4: Estimation of constant fault $\widetilde{M}_{\mathrm{cfm} 0}$ value by proposed FI system.

Faults were presented by changing the following values:

(i) Coulomb friction coefficient in reducing gear by $50 \%$ $\left(\widetilde{M}_{\mathrm{cfg} 0}=0.02 \mathrm{~N} \cdot \mathrm{m}\right.$ and $\left.\widetilde{M}_{\mathrm{cfg} 0}=0.02 \sin (0.5 t) \mathrm{N} \cdot \mathrm{m}\right)$ at the time from $t_{1}=5 \mathrm{~s}$ to $t_{3}=15 \mathrm{~s}$

(ii) Coulomb friction coefficient in DC motor by $50 \%$ $\left(\widetilde{M}_{\mathrm{cfm} 0}=0.02 \mathrm{~N} \cdot \mathrm{m}\right.$ and $\left.\widetilde{M}_{\mathrm{cfm} 0}=0.02 \sin (0.5 t) \mathrm{N} \cdot \mathrm{m}\right)$ at the time from $t_{2}=10 \mathrm{~s}$ to $t_{4}=20 \mathrm{~s}$

(iii) Electrical resistance $\widetilde{R}$ by $50 \%(\widetilde{R}=0.2 \Omega$ and $\widetilde{R}=$ $0.2 \sin (0.5 t) \Omega$ ) at the time from $t_{3}=15 \mathrm{~s}$ to $t_{5}=25 \mathrm{~s}$.

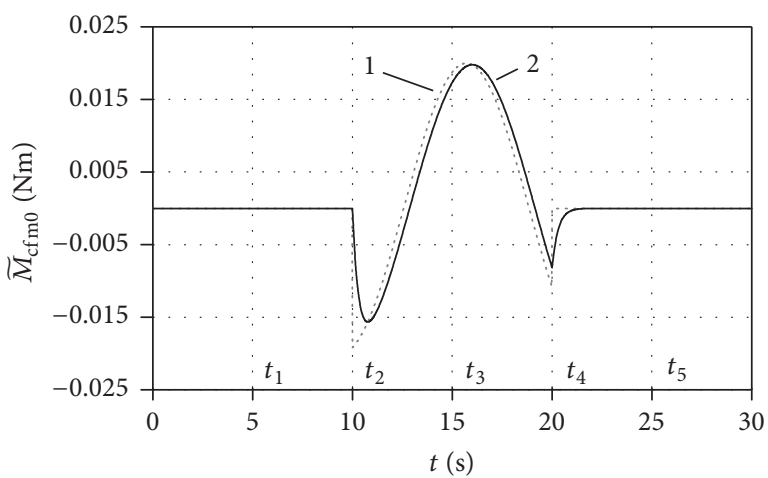

FIGURE 5: Estimation of variable fault $\widetilde{M}_{\mathrm{cfm} 0}$ value by proposed FI system.

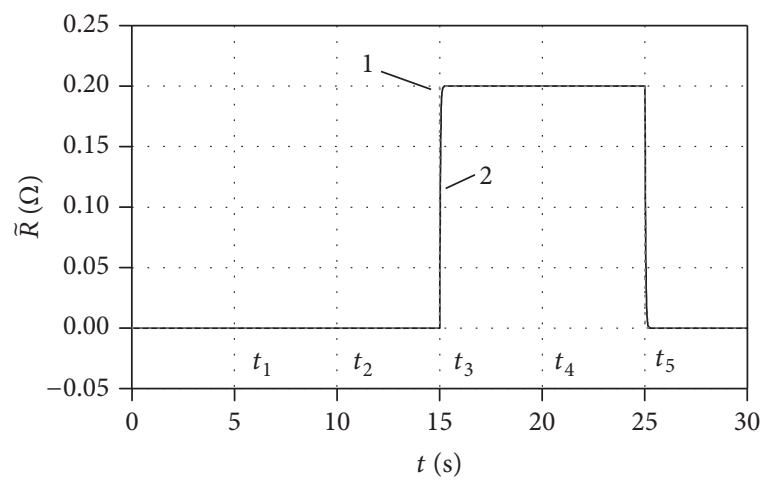

FIGURE 6: Estimation of constant fault $\widetilde{R}$ value by proposed FI system.

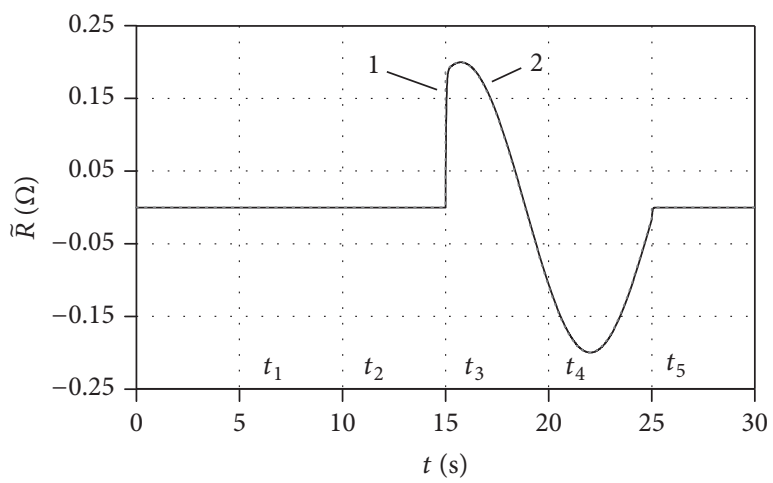

FIGURE 7: Estimation of variable fault $\widetilde{R}$ value by proposed FI system.

Values of introduced faults (curve 1) and estimated value of fault (curve 2) are shown in Figures 2-7. It follows from these figures that the constructed FI system provides accurate estimation of occurred fault. The results of simulation confirm the efficiency of the proposed method. Realization of the synthesized FI systems does not present any difficulties. These FI systems can be realized with the help of serial microprocessors. 


\section{Conclusion}

In this paper, the method of synthesis of accurate FI systems for electric servo actuators of the MM, which are described by nonlinear differential equations with variable parameters and partially measured state vector, is suggested. This method consists in applying the logic-dynamic approach for synthesis of diagnostic observers guaranteeing detection and localization of possible faults and introducing of special feedback in diagnostic observers, allowing identifying values of faults. Efficiency of the proposed method was confirmed by the results of simulation. The subject of further research is application of the proposed method for synthesis of the fault tolerant control systems for actuators of the MM.

\section{Conflicts of Interest}

The authors declare that they have no conflicts of interest.

\section{Acknowledgments}

These researches were supported by projects: Grant of President of Russia (MK-8536.2016.8) and Grants 16-29-04195 and 16-07-00300 of RFBR.

\section{References}

[1] M. Blanke, M. Kinnaert, J. Lunze, and M. Staroswiecki, Diagnosis and fault-tolerant control, Springer, Heidelberg, Third edition, 2016.

[2] P. M. Frank, "Fault diagnosis in dynamic systems using analytical and knowledge-based redundancy: a survey and some new results," Automatica, vol. 26, no. 3, pp. 459-474, 1990.

[3] F. L. Lewis, Robot Manipulator Control Theory and Practice, CRC Press, 2003.

[4] V. Filaretov, A. Zuev, D. Yukhimets, A. Gubankov, and E. Mursalimov, "The Automatization Method of Processing of Flexible Parts without their Rigid Fixation," Procedia Engineering, vol. 100, pp. 4-13, 2015.

[5] R. J. Patton, "Robust Model-Based Fault Diagnosis: The State of the ART," IFAC Proceedings Volumes, vol. 27, no. 5, pp. 1-24, 1994.

[6] R. Isermann and P. Balle, "Trends in the application of model based fault detection and diagnosis of technical processes," in Proc. of the 13th World Congress IFAC, p. 12, San Francisco, California, CA, USA, 1996.

[7] V. Filaretov, A. Zhirabok, A. Zuev, and A. Protcenko, "The new approach for synthesis of diagnostic system for navigation sensors of underwater vehicles," Procedia Engineering, vol. 69, pp. 822-829, 2014.

[8] V. Filaretov, A. Zuev, A. Gubankov, A. Procenko, and D. Yukhimets, "A new approach to synthesis of high speed and high reliable control systems for multilink manipulators," in Proceedings of the 2016 International Conference on Computer, Control, Informatics and its Applications (IC3INA), pp. 158-162, Tangerang, Indonesia, October 2016.

[9] R. Patton, P. Frank, and R. Clark, Issue of Fault Diagnosis for Dynamic Systems, Springer-Verlag, 2000.

[10] L. H. Chiang, E. L. Russell, and R. D. Braatz, Fault Detection and Diagnosis in Industrial Systems, Springer, 2001.
[11] F. Caccavale and L. Villani, Fault Diagnosis and Fault Tolerance for Mechatronic Systems:Recent Advances, Springer, Berlin, Heidelberg, 2003.

[12] V. F. Filaretov, "Synthesis of Adaptive Control Systems for Electric Servo Actuators of Manipulators," in Proceedings of the Proc. of the 18th DAAAM Int. Symp., Intelligent Manufacturing Automation, pp. 277-279, Zadar, Croatia, 2007.

[13] R. Seliger and P. Frank, "Fault diagnosis by disturbance decoupling nonlinear observers," in Proceedings of the Proc. of the 30th Conf. on Decision and Control, pp. 2248-2253, 1991.

[14] G. Schreier, J. Ragot, R. Patton, and P. Frank, "Observer design for class of nonlinear systems," in Proceedings of the Proc. of the IFAC Symposium SAFEPROCESS'97, pp. 498-503, Hull, UK, 1997.

[15] M. Staroswiecki and M. Comtet-Varga, "Fault detectability and isolability in algebraic dynamic systems," in Proceedings of the CD ROM Proc. of the European Control Conference ECC'99, Germany, Karlsrue, 1999.

[16] C. D. Persis and A. Isidori, "A geometric approach to nonlinear fault detection and isolation," IEEE Transactions on Automatic Control, vol. 46, no. 6, pp. 853-865, 2001.

[17] A. Shumsky and A. Zhirabok, "Nonlinear diagnostic filter design: algebraic and geometric points of view," International Journal of Applied Mathematics and Computer Science, vol. 16, no. 1, pp. 115-127, 2006.

[18] A. Zhirabok and S. Usoltsev, "Linear diagnosis methods for nonlinear systems," Automation and Remote Control, vol. 7, pp. 149-159, 2000.

[19] V. Filaretov, A. Zhirabok, A. Zuev, and A. Procenko, "Synthesis Method of Fault Tolerant Control System for Manipulators," Advanced Materials Research, vol. 717, pp. 551-556, 2013.

[20] V. F. Filaretov, A. N. Zirabok, A. V. Zuev, and A. A. Protsenko, "The Development of the Faults Accommodation System for Actuators of Multilink Manipulator," in Proceedings of the Proc. of the 23rd International DAAAM Symposium, pp. 575-578, Zadar, Croatia, 2012.

[21] L. Mironovsky, Functional diagnosis of dynamic systems, MSUGRIF, St, Petersburg, Russia, 1998.

[22] R. Isermann, Fault-Diagnosis Systems: An Introduction from Fault Detection to Fault Tolerance, Springer-Verlag, Berlin, Germany, 2006.

[23] M. Staroswiecki, H. Yang, and B. Jiang, "Progressive accommodation of aircraft actuator faults," in Proceedings of the IFAC Symposium SAFEPROCESS, pp. 877-882, Beijing, China, 2006.

[24] A. Siqueira and M. Terra, "A fault tolerant manipulator robot based on h2, hoo and mixed h2/ho markovian controls," in Proceedings of the IEEE International Conference on Control Applications, pp. 309-314, Taipei, Taiwan, 2004.

[25] V. Filaretov, A. Zhirabok, A. Zuev, and A. Protcenko, "Fault identification in nonlinear dynamic systems," in Proceedings of the 2016 5th International Conference on Systems and Control (ICSC), pp. 273-277, Marrakesh, Morocco, May 2016.

[26] A. Zhirabok, "Fault detection and isolation: linear and nonlinear systems," in Proceedings of the Proc. of the IFAC Symposium SAFEPROCESS97, pp. 903-908, Hall, UK, 1997. 


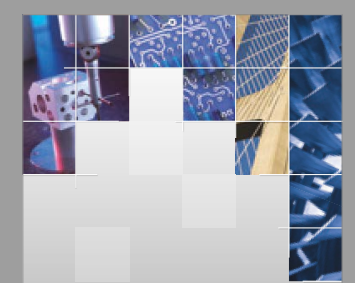

\section{Enfincering}
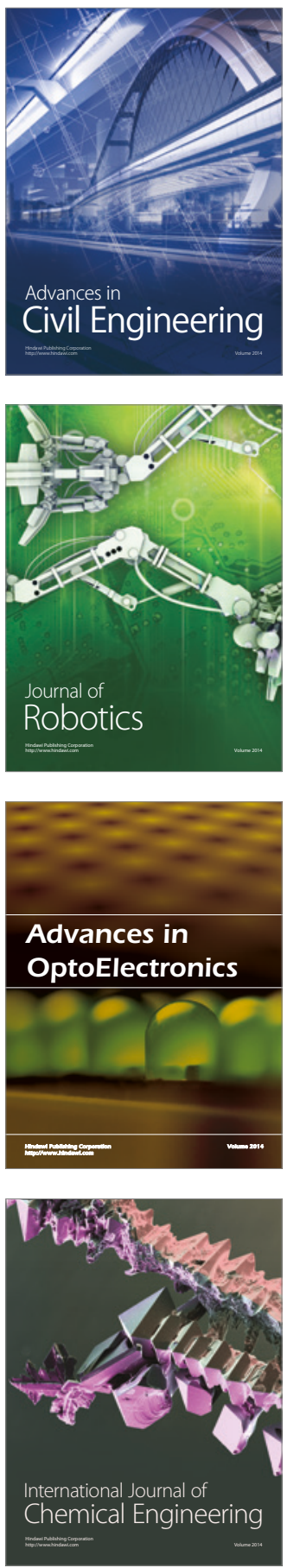

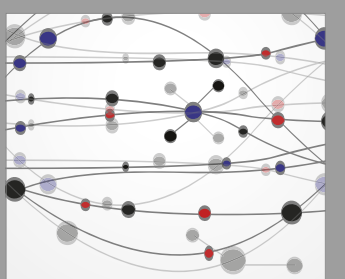

The Scientific World Journal

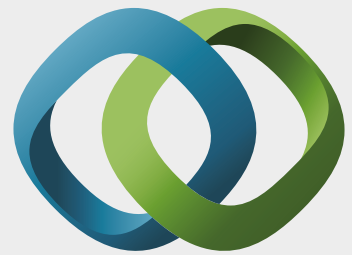

\section{Hindawi}

Submit your manuscripts at

https://www.hindawi.com
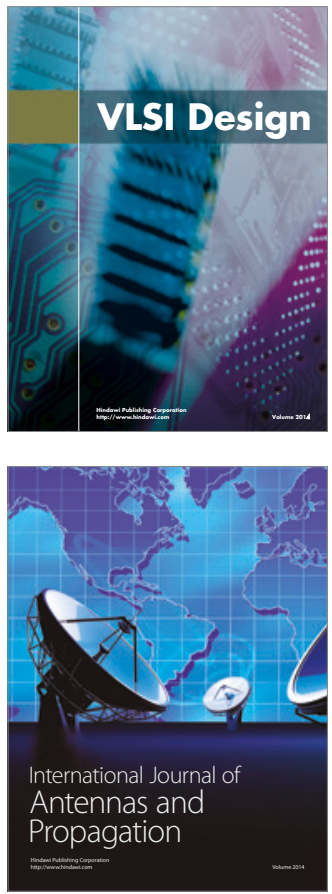

\section{Rotating}

Machinery
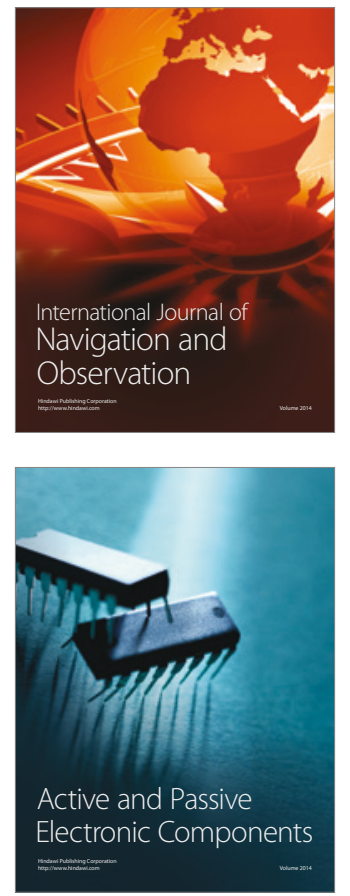
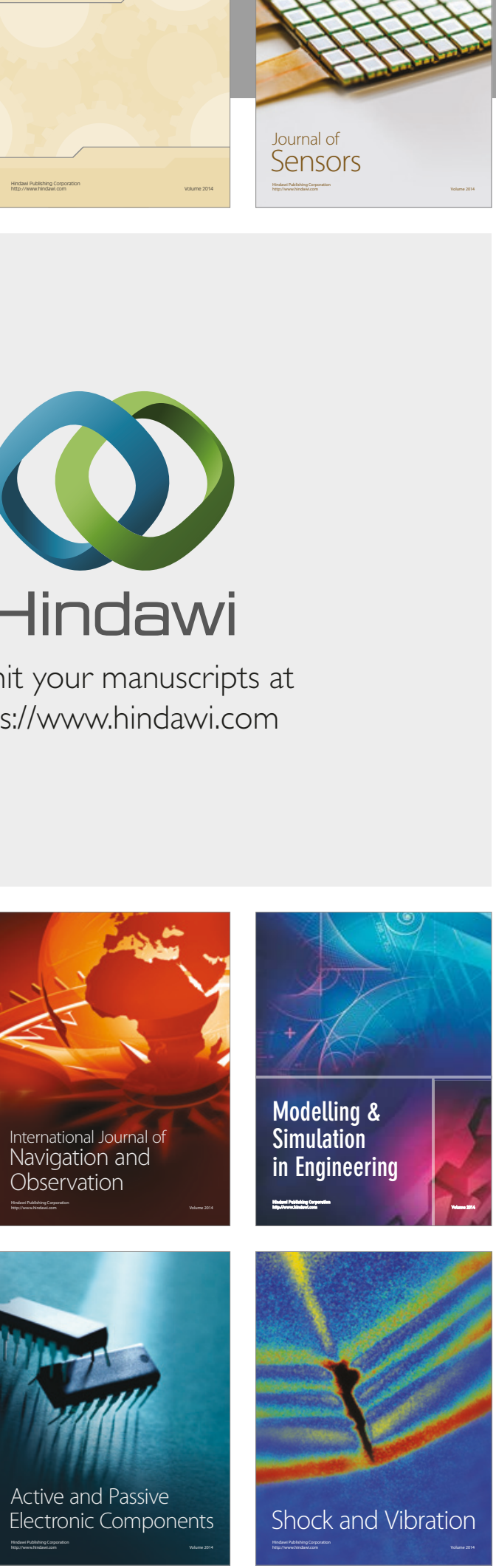
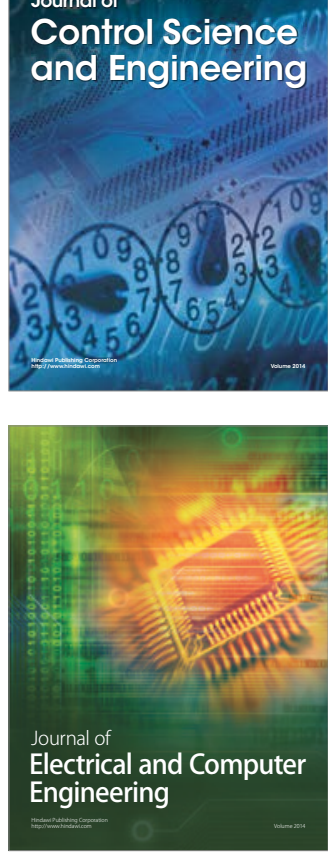

Distributed

Journal of

Control Science

and Engineering
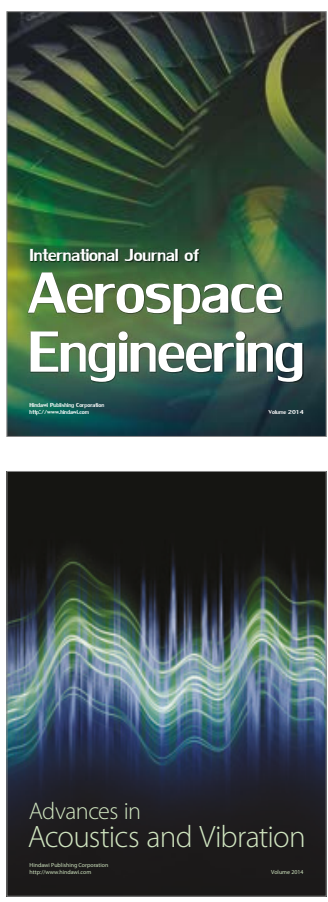

Sensor Networks 